\title{
AMIA: A Knowledge Representation Model for Computational Autobiographic Agents
}

\author{
Wan Ching Ho Scott Watson Kerstin Dautenhahn \\ Adaptive Systems Research Group \\ School of Computer Science, University of Hertfordshire, UK \\ \{W.C.Ho, S.E.J.Watson, K.Dautenhahn\}@Herts.ac.uk
}

\begin{abstract}
This paper describes a hierarchical design for knowledge representation in computational autobiographic memory. This model is named AMIA (Autobiographic Memory for Intelligent Agents). Inspired by research in human autobiographic memory from the areas of Psychology and Cognitive Science, the self memory system (SMS) is modelled as 1) the general knowledge structure of agents' significant events and 2) the unique "self" that SMS creates for an individual agent. We first carry out a concentrated review of autobiographic memory, emotion and event, and how they relate to each other. For AMIA, we start with an overview for different levels of the overall organisation and interactions between levels. We then focus on the design at the bottom level of AMIA - Action and Event levels. We also discuss the role of general event representations (GERs) which create knowledge representations for routine and highly expected events to be understood by autonomous agents, in conjunction with support from the autobiographic knowledge base in the reconstruction process of autobiographic events. Finally, we discuss the possible agent implementations for future research directions.
\end{abstract}

Index Terms - Knowledge representation, computational autobiographic memory, autobiograhpic agents, general event representations.

\section{INTRODUCTION}

When creating a cognitive model with the capabilities of learning and development for autonomous agents, memory and representation are essentially incorporated. Systems in earlier classical AI tend to represent knowledge in a machineunderstandable and symbolically-grounded way often for computers to understand human natural language and to perform efficient searches for problem-solving. Script, from Schank and Abelson [1], captures two important aspects of human memory in the perspective of developmental psychology: 1) it represents everyday events and activities, and 2) it has social-cultural components. However, early models like Frame [2] and Script represent knowledge in abstract static structures with little or no relation to "organic" developmental processes, or emotion.

\footnotetext{
${ }^{0}$ This work was partially supported by European Community (EC) and is currently funded by the eCIRCUS project IST-4-027656-STP. The authors are solely responsible for the content of this publication. It does not represent the opinion of the EC, and the EC is not responsible for any use that might be made of data appearing therein.
}

In recent years embodied AI emphasizes the on-going interaction between agents and their environments, in which the representation of the same object, depending on the behavioural or conceptual account for this object to an individual agent, can be very different and can lead to different meanings existing in agents' memory. Therefore, knowledge representation models for embodied autonomous agents, we believe, have to be ethologically-based [3]. Researchers in embodied AI have also suggested that these differences of meanings in knowledge representation among agents can be negotiated via social interaction [4] and social learning [5].

To create a comprehensive model for autonomous agents to represent knowledge of dynamic events devrived from the interaction with their environment and other agents' narrative storytelling; our approach is inspired by research from Psychology and Cognitive Science into autobiographic memory and human memory in general. Our model, AMIA (Autobiographic Memory for Intelligent Agents), does not aim to simulate all characteristics of adult autobiographic memory, but it captures essential features from a number of well developed psychological models. AMIA establishes the inter-relationship between the autobiographic knowledge base, which generates the working "self" based on the given goal activity, and general event representations (GERs) for handling routine and highly anticipated events for agents. External events, which can be experienced by agents, have been broken down into action units for the purpose of 1) comparing temporal action sequences with existing event categories in GERs and 2) attributing emotion to significant events which violate expectations generated by GERs.

\section{BACKGROUND}

In this section we carry out a concentrated review of interdisciplinary research on autobiographic memory from Psychology, Cognitive Science and Computer Science. We start by investigating the main cognitive and psychological model of the self memory system (SMS) proposed by Conway and Pleydell-Pearce [6]. We then investigate the subtle relationships between emotion and memory in order to identify the attribution of emotion to meaningful actions forming important events to be remembered by agents. 


\section{A. Human Autobiographic Memory}

Autobiographic memory is a specific kind of episodic memory [7] and contains significant and meaningful personal experiences of a human being. Two features of autobiographic memory are generally defined and accepted by researchers in psychology, as pointed out by [8]:

- Autobiographic memories are mental constructions of the self.

- They very often feature imagery while simultaneously containing abstract personal knowledge.

From the perspective of considering humans as social beings, developmental psychologist Nelson [7] carried out her investigations on how children develop their own autobiographic memory, and she suggested that the primary function of autobiographic memory is to allow the sharing of one's own memories with other people. Other studies in psychology and cognitive development have also pointed out the cognitive, as well as social, function of autobiographic memory as underlying all of human story telling and history-making narrative activity.

1) Self Memory System (SMS): Conway and PleydellPearce [6], introduced the self memory system (SMS) as a model of the relationship of autobiographic memory to the self. They suggested that the self is a set of memory structures which represent specific self-knowledge - knowledge which reflects to a specific self. There are three main components for SMS: 1) episodic memory system, 2) long-term self and 3 ) working self, as indicated by Conway and Pleydell-Pearce [4]. These components are mutually influening each other. The episodic memory system may provide input to the working self to fulfil its function of keeping track of plan execution in the course of ongoing goal processing. The working self, depending on goal activity status, mediates between episodic memory and the long-term self by generating a dynamic model of goal relevant knowledge and experience. The longterm self contains the knowledge required by the working self to organise and instantiate active goal processes.

2) Autobiographic Memory and Emotion: Memories with emotions can be seen as experiences of love, anger, happiness, and depression, etc. Psychological and Cognitive Science research have indicated that emotion plays an important role in both autobiographic memory encoding and retrieval processes. Conway [9] indicates that during the encoding process, emotion affects the vividness of events remembered in autobiographic memory: when experiencing an event with emotion, the cognitive system is more fully engaged in processing that event, in comparison to the processing of events which are not associated with strong emotional experience.

In the autobiographic memory retrieval process, a high level of emotional intensity is an important factor in the creation of a highly available (easy to recall) memory [9]. The reason is that, similarly to the memory encoding process, intense emotions lead to an increase in attention and more extensive processing of the associated event.

\section{B. Events}

In Developmental Psychology, it is widely acknowledged that the world is experienced by humans as a series of ongoing events. In general, events incorporate objects and relations in a larger whole, covering the basic knowledge representation structure as in traditional Cognitive Science research. Special aspects of events in knowledge representation are as follows [10]:

- They have a holistic structure which has strong characteristics for structural organisation in memory.

- They may change internally over time.

- Meanings are offered to individuals through everyday experiences.

- Stable mental elements, such as concepts and categories, are derived from initial contents (events) of mental representations.

Events are also very important in autobiographic memory because they provide a link from more abstract, thematic, goal-based autobiographic knowledge to representations of specific experience in episodes and event-specific knowledge [11]. Nevertheless, there are difficulties when utilising event structures to represent knowledge in agents' memory since they don't possess a cognitive structure to "understand" events naturally, as a human would. Modelling event structures in autobiographic memory involves three basic procedures including 1) breaking down an event into different units, 2) defining the meaning and importance that each unit contributes to the whole event, and 3) drawing a clear lines to mark the beginning and end of an event in memory.

The first two procedures have been experimentally modelled in agent research, such as Ho, et al [12] and Ho, et al [13]. For the third procedure, Conway, et al [11] indicated that the boundary of a current event in autobiographic memory is defined through the process of goal transitions - an event is ended when a goal is accomplished.

\section{AMIA (AUTOBIOGRAPHIC MEMORY FOR INTELLIGENT AGENTS}

In this section, for the purpose of modelling a simplified version of human SMS, we illustrate in detail the framework for knowledge representation in AMIA. To clearly identify features offered by components at the bottom level of the framework, after the introduction to the overall organisation, we divide the rest of this section into smaller subsections based on the structure and the role of each of these components.

\section{A. Overall Organisation}

With modelling certain features which reflect on the general characteristics of human autobiographic memory and 


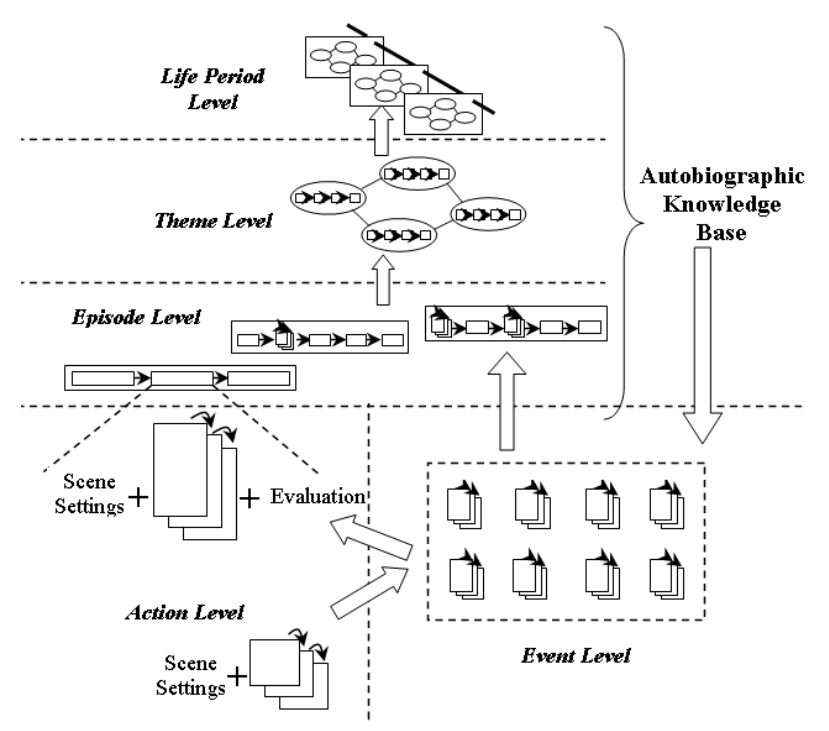

Fig. 1. An overview of knowledge representation in AMIA (details of some components are depicted in separate subsections with enlarged figures).

GER, AMIA covers different aspects of information processing for believable and intelligent agents. Figure 1 shows an overview for the whole knowledge representation framework of AMIA.

AMIA consists of five main components: Action Level, Event Level, Episode Level, Theme Level and Life Period Level. They are organised in a bottom up fashion - high level components are constructed by contents from within the component located at the next level beneath them. The duration of these separate components differs according to the level of granularity involved in their definition.

\section{B. Action Level}

As we argued in the Background section an event, which has some particular characteristics such as its holistic structure and internal dynamics, is very suitable to be used for modelling knowledge representation in autobiographic memory. Here we define an event, in AMIA, as a collection of any number of actions which can be grouped together based on their inclusion in a specific psychologically meaningful pattern. Furthermore, a general event experienced by an agent should have basic components that include:

- Scene settings - time, location, agents that involved into the event, etc.

- Situation-action pattern - a sequence of actions that agent performed individually over time under specific situations.

Figure 2 illustrates basic components of an event and how an event can be absorbed by a GER. For events which are novel or significant, an agent's current emotion may be attributed to related actions when constructing these events in the Episodic Level of AMIA. At the same time, evaluations

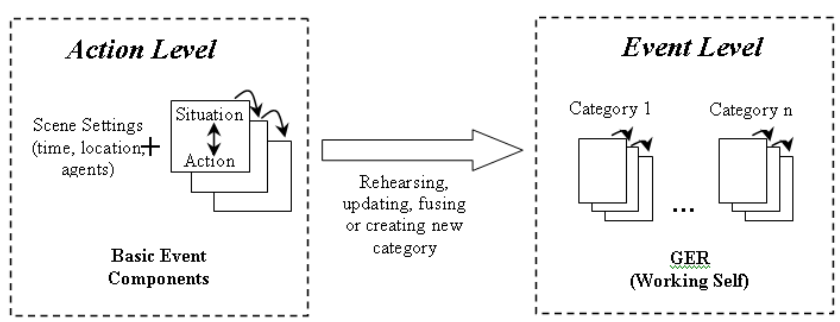

Fig. 2. Basic event components and the relationship between Action Level and Event Level.

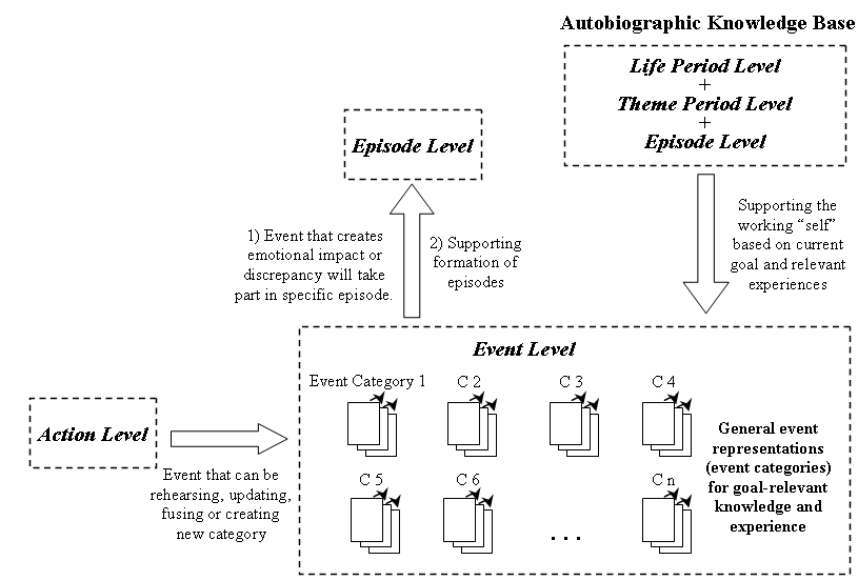

Fig. 3. GERs in Event Level and how they interact with other components in AMIA.

may also be built up and attached to the event structure. This feature will be discussed in the last subsection.

\section{Event Level}

Each event experienced by an agent will be examined through a "filtering" process in the Event Level. A new event category is created when an event cannot be absorbed into existing GERs - the event is different from existing GERs - a novel event. Forming the existing GERs is a critical and dynamic process in maintaining the coherent behaviours of agents and it requires retrieving a considerable amount of knowledge from autobiographic memory based on the current ongoing goal activity. Novel and significant events, which may create a considerable amount of discrepancy between the standard and current state of the world, will provide event specific knowledge (ESK, see next sub-section Episode Level for details) and take part in forming particular episodes in the Episode Level. For example, in previous work [12] significant events were indicated by a substantial amount of changes in agents' internal physiological variables. Moreover, GERs also provide categories of routine events to support the constructions of important episodes. These features are illustrated in Figure 3.

1) GERs: In AMIA, GERs are event categories organised dynamically based on the current goal activity. Their 
goal-oriented event categories can be perceived as memory schemata and scripts since they generally encapsulate all of an agent's knowledge of a particular type of object, situation or event. Knowledge for digesting routine events - events that are familiar and do not create emotional impact to the agent - is similar to human semantic knowledge which is used to understand the world. Therefore these event categories provide expectations about what the agent will experience (e.g. see, hear).

When an event from the Action Level is evaluated against GERs, if it does not differ from the existing categories, it will be "absorbed" into the GERs through rehearsing the categories and its content details can decay overtime. Therefore routine events do not have great details in GERs but their schematic structure and meaning are preserved.

If the event is novel or some part of it violates the expectations provided by GERs, it will be encoded as a new category and also, if it contains procedural information such as if it exists as part of a sequence of events for a common goal, this event will also be encoded into episodes in the higher Episode Level.

2) Goal Structure and Working Self: Agents' specific autobiographic memories are constructed from event specific knowledge (ESK) which forms memories in Episode, Theme and Life Period Levels [6]. This construction takes place in the conjunction of the working "self" with the autobiographic knowledge base (as illustrated in Figure 3) and occurs when the complex goal system of the working self modulates knowledge access and memory construction. Therefore the working "self" for agents can be seen as a subset of working memory control processes closely connected with the current goal structure and it always monitors the goal accomplishment.

The goal structure modulated by the working "self" is designed to form an iterative loop whose purpose is to reduce the discrepancy between desired and actual goal states. In performing this discrepancy reduction, behaviour is regulated. Goals (and their respective sub-goals) emerge from autobiographic knowledge and guide selective attention and the behavioural processes necessary to perpetuate the current goal structure.

In order to ensure this structure is maintained, the working "self" supported by the autobiographic knowledge base also continuously monitors events and verifies the current goal attainment. In this way, the goal structure directs behaviour in a manner which compliments the agent's previous understanding of the situation. In turn, this verification process will lead to the encoding and further consolidation of GERs - presumably in order to reduce the quantity of information that actually has to be stored and to facilitate a more cohesive "self".

In summary, the autobiographic knowledge-base and the working "self" for agents are instrumental in the genera- tion and maintenance of a goal structure, thus providing a cohesive narrative for the agent to follow. After presenting the general framework of the relationship of goal activity to memory in the working self, we now turn to event specific knowledge (ESK) in the Episode Level.

\section{Episode Level}

When an event, or at least part of it, violates an agent's goal-related expectations and cannot be "absorbed" by the existing GERs, some temporal information and details of specific actions which create emotional impacts from this event will remain as event specific knowledge (ESK) in the Episode Level. Thus, episodes are represented in the autobiographic knowledge base largely by event-specific knowledge, and an event is a representation that binds together a set of significant-action-based ESK.

In order to ensure that each episode in autobiographic memory is meaningful and coherent, GERs from the Event Level generate routine events and semantic knowledge (e.g. information for the sequence of some typical events in a specific episode) to support the formation of a complete episode. Note that this process is also influenced by the autobiographic knowledge base which constructs the working "self" for the agent based on the goal activity.

Figure 4 shows a general picture of contents in the Episode Level, interactions between the Episode Level and other components in the framework, and details of a significant event as an example.

Events retained in the Episode Level and forming specific episodes are different in duration and complexity and they are highly specific unique experiences. Unlike routine events in GERs, they also act as organising representations for memories of more specific occurrences. Therefore knowledge constructing these specific events constitutes a central feature in autobiographic memory.

\section{E. Theme and Life Period Levels}

On top of the Episode Level, in AMIA we propose Theme and Life Period Levels as more abstract levels of autobiographical knowledge that can further characterise an individual agent's life.

Theme structures would be constructed using components from multiple episodes which typically consist of several events and fewer themes than episodes will exist for agents because of the structural nature of both in time. Thus significant events and episodes are representations that provide the "data" that ground themes and goals in actual or recorded experience. The Life Period Level contains life periods and each of them is constructed by themes in temporal sequences based on the goal-oriented working "self" the agent had in a relatively longer period of time.

The autobiographic knowledge base consists of the levels of Episode, Theme and Lifetime Period that structure the episodic memory system to generate specific autobiographic 


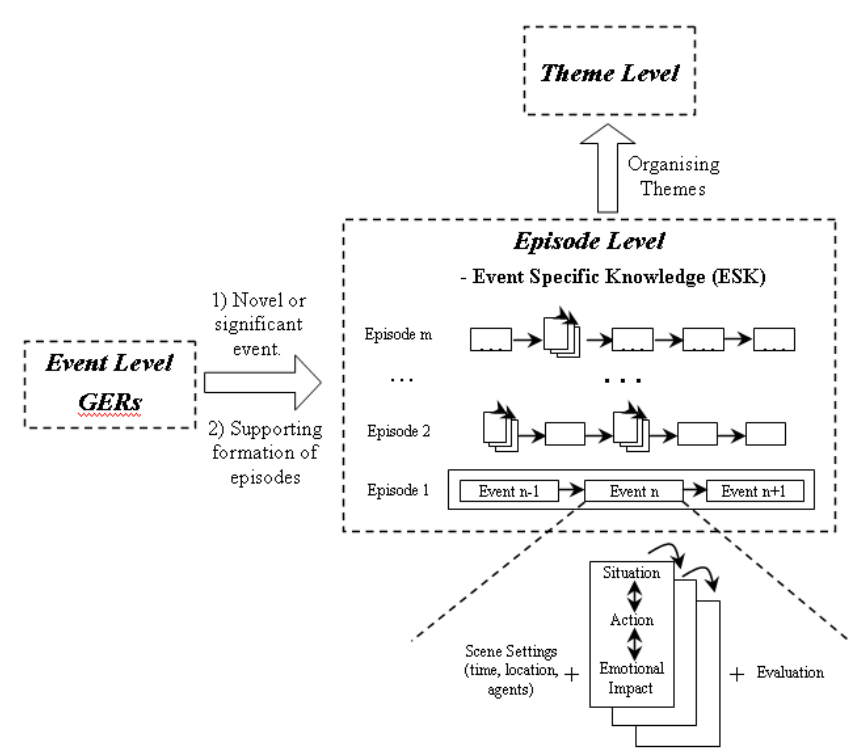

Fig. 4. Event specific knowledge (ESK) in Episode Level and how it forms episodes.

memories. For example, as the autobiographic agent changes its environment, role in the system, or relationship with users or other agents; its current psychological representation of past experiences gradually transforms into self-knowledge in the Theme and Lifetime Period Level. The period of transition may reflect the re-organization of the working "self" which is in charge of goal structure in any given time.

\section{F. Evaluation of Different Levels in the Framework}

Each component of the knowledge representation structure in AMIA (Life Period, Theme, Episode, Event, and Action) can provide an evaluation attaching to its contents separately. Evaluations for routine actions and events in Action and Event Levels are encoded in the GER structure - because these actions and events are highly predictable and there is no violation of the agent's expectations.

Evaluations for components with more abstracted contents (Episode, Theme and Life Period Levels) will be created during the first reflective process, and provide an overall summary of the experiences that belong to the actions/events that are included in the specific episode, for example, a conversational autobiographic agent remembering the interaction with an individual user in a fixed period of time. This example as a whole (from the user introducing himself/herself to saying good-bye to the agent at the end) constitutes a specific episode, which will be part of the theme "interacting with user X".

The particularly memorable events that occurred during the interaction with user $\mathrm{X}$ (such as $\mathrm{X}$ saying some incomprehensible words or telling the agent about his happy experiences) are all encoded with an accompanying emotional impact and evaluation. For example, in the system input interface, users can have different text symbols (e.g. exclamation marks and smiling-face icons) and colours (e.g. red and blue colours represents angry and happy respectively) for expressing their emotions attributed to memorable events which can be comprehended by the agent. Since, in this way, the agent can remember each of these particular events from user X's input, it can reflect on the interaction as a whole in the Episode Level and give an overall impression of how it feels about user X.

However, this reflection can also be influenced by the future experiences stored in the autobiographic knowledge base; in that case evaluations and further re-evaluations may change as the "self" of the agent develops. Extending the same example, currently our conversational agent may generate a general impression of user X as "a positive person" following the interaction with him/her; later after the agent had conversations with another fifty users, the impression of user X may only be "a normal person with some happy experiences". This effect is the result of categorisation process in GERs since user X's individual happy experiences have been repeated by other users' experiences perceived by the agent and they are no longer unique and significant.

\section{Discussions of the Implementation for Agents}

With autobiographic knowledge, agents can organise significant experiences remembered throughout their "lifetime" and develop their unique perspectives towards different objects or events things. With a simplified version of autobiographic memory and semantic world knoweldge, we have realised the concepts similar to the Action and Event levels proposed in our previous work [13]: An educational antibullying software populated with autonomous 3D characters. With the simplified version of autobiographic memory, agents as reported in [13] can 1) have more natural and appropriate emotional reactions to events, and 2) tell a user of their past experiences as a personal narrative. Therefore we expect that a comprehensive model of autobiographic memory like AMIA can further increase the believability of autonomous and possibly conversational agents.

As researchers in computational event memory models have claimed that autobiographic memory encounters testing difficulties due to its effortful memory retrieval [14], AMIA has transformed the structural hierarchy of the representation of autobiographic knowledge into an implementable computational model which is suitable for believable and intelligent agents.

Abstracted autobiographic knowledge in higher levels of the framework - Theme and Life Period levels - may not be applicable to agents solving quick problems, e.g. answering users' short questions in a limited period of time. However, we believe it is important to have these two abstract levels of knowledge for an agent to avoid exhaustive searches when it tries to generate a general summary of past events. Instead 
of searching and comparing low-level action-situation pattern details, agents can instantiate a theme using the existing autobiographic knowledge in the Theme Level. Moreover, it is also evident that humans abstract high-level knowledge and rules from their experiences and can detect the emergence of similar meanings in other experiences [14].

Narrative computational agents are usually preprogrammed either with temporal and structured stories or with simultaneously selecting story sequences from a large story database when they are interacting with human users in storytelling systems. Therefore, they can take advantage of mechanisms used in natural historically grounded systems [15] and enhance the friendliness of these systems. While appreciating the success narrative agents bring to storytelling systems or software interfaces, the investigation of how agents themselves can benefit from autobiographic narrative - using their existing experiences to interpret a new story - is missing in the Narrative Intelligence research field. A new story is defined here as an agent perceiving a new event itself or receiving a story from another agent through communication. An agent should then be able to form a unique psychological interpretation of this story by drawing upon their own existing autobiographic knowledge.

Another main research issue in character-based narrative storytelling systems, i.e. role-play dramas or games, is how to provide good "actors" - agents that react to different situations in stories with appropriate emotional expression. We argue that rather than setting a fixed group of parameters which instruct agents how to "act" in a particular situation, it is more realistic and flexible to shape agents' "personality" in a story and determine their emotion in a given situation by allowing them to have their own autobiographic knowledge. This approach thus 1) allows for the creation of agents which can be "born with" certain types of personality for role-play stories, e.g. via an authoring tool, each agent is generated with certain cohesive autobiographic knowledge to enhance its readiness for acting a specific role; and 2) supports long-term development of virtual actors as they gain more experience from acting in each new situation.

\section{CONCLUSION}

In AMIA, we define the autobiographic knowledge base as significant events which contain novel and mostly unique sensory experiences for agents. As a foundation of that, we specify low-level basic units for event knowledge which include basic scene setting and situation-action patterns in temporal sequences. We also address the importance of the dynamically reconstructed working "self" based on the agent's current goal activity for its influences on forming general event representations to digest ongoing events.

We have adopted a hierarchical structure in AMIA which illustrates that agents can benefit from autobiographic knowledge in terms of learning from past experiences and long-term development. On a behavioural level, we reviewed that, with autobiographic memory, agents' adaptivity can be enhanced. Furthermore, we discussed that autobiographic knowledge can ground higher level cognitive processes, e.g. goal formulation, attention and emotion - resulting in increased agent believability. Finally, we discussed possible future directions for implementing AMIA in believable and intelligent agents.

\section{ACKNOWLEDGMENT}

The authors would like to thank Prof. Chrystopher L. Nehaniv, for his assistance with our previous research into autobiographic agents.

\section{REFERENCES}

[1] R. C. Schank and R. P. Abelson, Scripts, Plans, Goals and Understanding: An Inquiry into Human Knowledge Structures. Hillsdale, New Jersey: Lawrence Erlbaum Assicuates, 1977.

[2] M. Minsky, "A framework for representing knowledge," in The Psychology of Computer Vision, P. H. Winston, Ed. New York: McGrawHill, 1975, pp. 211-277.

[3] K. Nelson, "Event representation, narrative development, and internal working models," Attachment and Human Development, vol. 1, pp. $239-251,1999$.

[4] K. Dautenhahn and T. Christaller, "Remembering, rehearsal and empathy - towards a social and embodied cognitive psychology for artifacts," in Two sciences of the mind. Readings in cognitive science and consciousness, S. O'Nuallain and P. McKevitt, Eds. John Benjamins North America Inc, 1996, pp. 257-282.

[5] L. Steels, "Intelligence with representation," Philosophical Transactions: Mathematical, Physical and Engineering Sciences, vol. 361, no. 1811, pp. 2381-2395, 2003.

[6] M. A. Conway and C. W. Pleydell-Pearce, "The construction of autobiographical memories in the self memory system," Psychological Review, vol. 107, pp. 261-288, 2000.

[7] K. Nelson, "The psychological and social origins of autobiographical memory," Psychological Science, vol. 4, pp. 7-14, 1993.

[8] M. A. Conway, C. W. Pleydell-Pearce, and S. E. Whitecross, "The neuroanatomy of autobiographical memory: A slow cortical potential study (scp) of autobiographical memory retrieval," Memory and Language, vol. 45, pp. 493-524, 2001.

[9] M. A. Conway, Autobiographical Memory: An Introduction. Buckingham: Open Univ. Press, 1990.

[10] K. Nelson, Event knowledge and cognitive development. London: Lawrence Erlbaum Associates, 1986, pp. 1-19.

[11] M. A. Conway, S. J. A., and T. A., "The self and autobiographic memory: correspondence and cohenrence," Social Cognition, vol. 22, no. 5, pp. 491-529, 2004.

[12] W. C. Ho, K. Dautenhahn, and C. L. Nehaniv, "A study of episodic memory-based learning and narrative structure for autobiographic agents," in Proceedings of Adaptation in Artificial and Biological Systems, AISB 2006 conference, vol. 3, 2006, pp. 26-29.

[13] W. C. Ho, J. Dias, R. Figueiredo, and A. Paiva, "Agents that remember can tell stories: Integrating autobiographic memory into emotional agents," in Autonomous Agents and Multiagent Systems (AAMAS). ACM Press, 2007, accepted.

[14] M. A. Conway, "Autobiographical memories and autobiographical knowledge," in Remembering our past: Studies in autobiographical memory, D. C. Rubin, Ed. Cambridge, UK: Cambridge Univ. Press, 1996, pp. 67-93.

[15] C. L. Nehaniv, "Narrative for artifacts: Transcending context and self," in Narrative Intelligence, ser. AAAI Fall Symposium 1999. AAAI Press, 1999, pp. 101-104, Technical Report FS-99-01. 